\title{
Anemia Among Unbooked Parturients With Asymptomatic Malaria Parasitemia At A Tertiary Institution Southeast Nigeria.
}

\author{
Nwali MI, Umeora OUJ, Ozumba BC, Onoh RC, , Ezeonu PO, Agwu UM. \\ ${ }^{I}$ federal Teaching Hospital, Pmb 102, Abakaliki, Ebonyi, Nigeria. \\ 2 university Of Nigeria Teaching Hospital, Ituku-Ozalla, Enugu, Nigeria.
}

\begin{abstract}
Background: Anemia in pregnancy is common in malaria endemic areas like ours and is a risk factor for maternal morbidity and mortality. Its consequences can be prevented by instituting adequate preventive measures.

Objective: The aim of this study is to determine the prevalence of anemia among unbooked women presenting in labour at term and its association with asymptomatic malaria parasitemia.

Methods: A prospective cross-sectional study conducted in the labor ward complex of the Federal Teaching Hospital Abakaliki over three months period from March-May 2012. Two hundred and fifty unbooked pregnant women who presented in labor were consecutively recruited after an informed consent. A structured data sheet was administered to each parturient. The hemoglobin concentration was determined using the Hemocue system. Analysis was done using Epi info programme (2005) of CDC Atlanta USA 3.3.2 version. Logistic regression was used to determine level of associations. Level of significance was set at $P$-value $<0.05$.

Results: A total of 250 parturients participated in the study. The mean age in the study was $28.2 \pm 7.2$ years and ranged from $19-45$ years. The mean parity was $2 \pm 1.7$ and ranged from $0-5$. One hundred and seven parturients (42.8\%) were primigravida while $143(57.2 \%)$ were parous women. Majority of the parturients were of low socio-economic class. The prevalence of asymptomatic malaria parasitemia was 194 (77.6\%). The prevalence of anemia in the study was $46.8 \%$. There was significant association between asymptomatic malaria parasitemia and maternal anemia with $P<0.001$. None of the parturients without malaria parasitemia had anemia.
\end{abstract}

Conclusion: Anemia in pregnancy was common among unbooked pregnant women. It was also common among parturients with asymptomatic malaria parasitemia.

Keywords: Anemia, Asymptomatic malaria parasitemia, pregnancy, unbooked

\section{Introduction}

Each year, approximately 50 million women living in malaria endemic area worldwide become pregnant, of which more than half live in tropical Africa with intense Plasmodium falciparum transmission. ${ }^{1}$ An estimated 10,000 of these women die as a result of malaria infestation and more than half of these deaths are due to anemia resulting from malaria. ${ }^{1} \mathrm{Up}$ to $20 \%-58.4 \%$ of asymptomatic parasitemia in pregnant women has been reported with anemia as a major complication., ${ }^{2,3}$ Optimal hemoglobin level is important for both maternal and fetal well being during pregnancy. Seventy percent of pregnant women in Nigeria suffer malaria. ${ }^{4}$ There is inevitable subclinical hemolysis associated with asymptomatic malaria parasitemia which contributes significantly to the almost universal anemia found in pregnant women in the tropics. ${ }^{2}$ There was a report of increased destruction of both parasitized and unparasitized red blood cells during malaria infestation. ${ }^{5}$ Apart from hemolysis, asymptomatic malaria parasitemia has been associated with decreased iron absorption from the gastrointestinal tract. ${ }^{6,7}$ Suggestions has been made for maternal anemia to be used for surveillance of malaria control in malarious sub- Saharan Africa. ${ }^{8}$ It has been reported that prevalence of severe anemia attributable to malaria among pregnant women of all gravidities is $26 \%$ and that timing of infection during pregnancy had no effect on maternal anemia. ${ }^{9}$ The WHO criterion of hemoglobin concentration less than $11.0 \mathrm{~g} / \mathrm{dl}$ was used in this study as a universal standard for anemia not to underrate the burden of anemia in pregnancy in our environment. The report from Calabar showed 59.6\% prevalence of anemia in pregnancy among asymptomatic malaria parasitemic clients at their first antenatal visit. ${ }^{10}$

This study is aimed at determining the prevalence of anemia among unbooked parturients as well as the association of anemia in pregnancy and asymptomatic malaria parasitemia in labour at Federal Teaching Hospital Abakaliki. This will show the enormity of the problem, create awareness and help to scale up preventive measures against malaria in pregnancy to reduce the morbidity and mortality associated with this in the Obstetric community. 


\section{Materials And Methods}

This prospective cross-sectional study was conducted in the Federal Teaching Hospital, Abakaliki, Ebonyi state, Nigeria over a period of three months from March to May 2012. Federal Teaching Hospital is the major tertiary hospital in the state, located in the capital city, Abakaliki, Southeastern Nigeria. The state has a population of 2.1 million people as was reported in 2006 by the National Population Commission. Malaria transmission is holoendemic in the state. The study population included all the unbooked pregnant women presenting in labor at term within the study period. The exclusion criteria were those who refused consent, preterm labor, multiple gestations, HIV in pregnancy, antepartum hemorrhage and those with symptomatic infection.

A structured data sheet was administered to the women by the authors after explaining the purpose of the study to them and obtaining an informed consent which was re-confirmed in the postnatal ward after delivery. Information obtained included age, parity, gestational age and social class. After aseptic procedure, a sterile syringe was used to collect 5 milliliters of blood from the antecubital vein. This was preserved in a prelabeled EDTA bottle for each parturient for estimation of hemoglobin concentration. A thick and thin blood films were also prepared on two labeled glass slides for each parturients.

The hemoglobin estimation was done using the hemocue system (hemocue AB, Angel-holm Sweden) which consist of a pre-calibrated, portable, battery or main operated photometer. No dilution was required as blood was run by capillary action directly into a corvette containing sodium nitrite and sodium azide that convert the hemoglobin to azidemethemoglobin. The absorbance is then measured at a wave length of 565 and $880 \mathrm{Nm}$. This is reliable as high level of bilirubin, lipids or white blood cells do not affect measurements. ${ }^{11}$ The blood films were stained with 5\% Giemsa stain for twenty minutes after which it was rinsed with water air dried and microscopic examination done under oil immersion at 100x magnification.

Anemia was determined using the WHO standard of hemoglobin concentration less than $11.0 \mathrm{~g} / \mathrm{dl}$. Anemia was classified as mild at hemoglobin concentration of $8-10 \mathrm{~g} / \mathrm{dl}$, moderate at hemoglobin concentration of $6-8 \mathrm{~g} / \mathrm{dl}$ and severe at hemoglobin concentration of $\langle 6 \mathrm{~g} / \mathrm{dl}$. In the slides reading, parasite density of $1-10 / 0.25 \mu \mathrm{L}$ of blood or more was regarded as positive. Those affected were managed according to Obstetrics and Gynecology departmental protocol. The samples were prepared read and analysed by each of two experienced laboratory scientists dedicated to the study to ensure quality control.

The Research and Ethics Committee of the Federal Teaching Hospital approved the study protocol. The minimum sample size of 206 unbooked women for the study was calculated using $16 \%{ }^{12}$ prevalence from a similar study in the state with the formula for sample size calculation proposed by Daniel, Lwanga and Lameshow. ${ }^{13,14}$ The coded data was fed into the computer using Epi info ${ }^{\mathrm{TM}}$ programme (2005) of CDC Atlanta USA 3.3.2 version and analysis done. Logistic regression was used to determine level of associations while keeping the confounding variables constant. Level of significant was set at P-value less than 0.05 .

\section{Results}

A total of 250 parturients participated in the study. The prevalence of asymptomatic malaria parasitemia in the study was $77.6 \%$. A total of $117(46.8 \%)$ parturients had anemia in the study. The mean age of participants in the study was $28.2 \pm 7.2$ years and ranged from $19-45$ years. The mean parity of participants in the study was $2 \pm 1.7$ and ranged from para 0 to para 5 . Majority of the parturients were of low socioeconomic class (class $4 \& 5$ ). None of the parturients without parasitemia had hemoglobin concentration $<11.0 \mathrm{~g} / \mathrm{dl}$. Among the 194 parasitemic parturients, $117(60.3 \%)$ had anemia. These were illustrated in Table 1.

One hundred and seventy six (89.8\%) had parasite density of one plus (+) out of which $43.8 \%$ had hemoglobin concentration of $\geq 11.0 \mathrm{~g} / \mathrm{dl}, 53.4 \%$ had mild anemia and $2.8 \%$ had moderate anemia. Among the parturients with two plusses (++) of parasite density, none had hemoglobin concentration $\geq 11.0 \mathrm{~g} / \mathrm{dl}$. This was statistically significant $\left(x^{2}=31.735\right.$, $\mathrm{P}$ value $\left.<0.001\right)$. No parturient had severe anemia. This was illustrated in Table $2 \& 3$

The correlation between malaria parasitemia and hemoglobin concentration was tested using logistic regression and the result showed a negative coefficient of -1.329 , standard error of 0.356 , F-test of 13.9565 and a $\mathrm{P}$ value of 0.000246 . This showed a strong correlation between anaemia in pregnancy and malaria parasitemia.

\section{Discussion}

Anemia in pregnancy is an important cause of maternal morbidity and mortality directly or indirectly. Malaria in pregnancy is an under-recognized cause of anemia in endemic areas as it is usually asymptomatic. ${ }^{15}$ The high prevalence of asymptomatic malaria parasitemia in this study shows the enormity of the problem. This prevalence of $77.6 \%$ however is lower than $95.4 \%$ reported in Calabar. ${ }^{16}$

The prevalence of anemia among the parturients that actually had malaria parasitemia as deduced from was $60.3 \%$. This compares with the report of $59.6 \%$ prevalence from Calabar but lower than $71 \%$ reported in Lagos. ${ }^{10,15}$ Anemia may be due to hemolysis as a result of malaria parasitisation. There is also increased 
destruction of both parasitized and unparasitized red blood cells in the spleen during malaria infestation. ${ }^{5}$ Asymptomatic malaria parasitemia has also been associated with decreased absorption of iron from the gastrointestinal tract. ${ }^{6,7}$

The study demonstrated strong negative correlation between asymptomatic malaria parasitemia and maternal hemoglobin concentration which was significant. Anemia also increased with parasite density in the study. This buttresses the impact of malaria parasitaemia on the level of haemoglobin resulting in anemia in pregnancy.

\section{Conclusion}

This was high prevalence of anemia among unbooked parturients. Anemia in pregnancy was common among paturients with asymptomatic malaria parasitemia and the level of anemia was directly related to the level of malaria parasite density.

\section{Recommendations}

There is need to scale up preventive measures (ITN, IPT and effective treatment) against malaria in pregnancy due to the observed impact of asymptomatic malaria parasitemia.

Vector control measures should also be put in place to reduce the level of malaria transmission in our environment.

\section{Conflict of interest}

There is no conflict declared.

\section{References}

[1]. World Health Organization. Malaria: high risk groups/pregnancy. Accessed August 15, 2011.

[2]. Okpere EE. Malaria in pregnancy. In: Okpere EE (ed). Clinical Obstetrics. Revised edition. UNIBEN Press. Benin. 2004. Pp 56-61.

[3]. Nwagha UI, Ugwu VO, Nwagha TU and Anyaehie USB. Asymptomatic plasmodium parasitemia in pregnant Nigerian women: almost a decade after Roll Back Malaria. Trans R Soc Trop Med Hyg. 2009; 103 (1): 16-20.

[4]. Federal Ministry of Health. National malaria control programme in Nigeria. Roll Back Malaria Annual Report. Abuja. 2005; Pp 8-9.

[5]. Olugbenga AM et al. Malaria at parturition in Nigeria: Current Status and Delivery Outcome. Infectious disease in Obs and Gynae. 2009; 2009: 7 pages. DOI:10.1155/2009/473971.

[6]. Cercamondi CI, Egli IM, Ahouandjinou E, Dossa R, Zeder C, Salami L. et al. Afebrile plasmodium falciparum parasitemia decreases absorption of fortification iron but does not affect systemic iron utilization: A double stable isotop study in young Beninese women. AM J Clin Nutri. 2010; 92 (6): 1385-1392.

[7]. Savage EJ, Msyamboza k, Gies S, Alessandro UD and Brabin BJ. Maternal anemia as an indication for monitoring malaria control in pregnancy in sub-Saharan Africa. BJOG 2007; 114: 1222-1231.

[8]. Kalilani L, Mofolo I, Chaponda M,Rogerson SJ and Meshnick SR. The effect of timing and frequency of plasmodium falciparum infection during pregnancy on the risk of low birth weight and maternal anemia. Trans R Soc Trop Med Hyg. 2010 ; 104 (6): $416-$ 422 .

[9]. Schantz-Dunn J and Nour NM. Anemia in pregnancy. Rev Obstet Gynecol 2009; 2(3): 186-192.

[10]. Agan TU, Ekabua JE, Udoh AE, Ekanem EI, Efiok EE and Mgbekem MA. Prevalence of anemia in women with asymptomatic malaria parasitemia at first antenatal visit in UCTH Calabar. Int J Women's Health.2010; 2: 229-233.

[11]. Dacie and Lewis. Practical hematology, Lewis son, Bain BJ, Dates I (eds). $9^{\text {th }}$ Edition, Churchill and Livingstone, London. 2001: Pp 23.

[12]. World Health Organization. Lives at Risk: Malaria in pregnancy, 2003; information sheet. Retrieved March $30,2011$.

[13]. Daniel WW. Biostatistics; A foundation for analysis in the health sciences. $7^{\text {th }}$ edition, John Wiley and sons. New York. 1999.

[14]. Lwanga SK and Lameshow S. Sample size determination in health studies: A practical manual. Geneva. World Health Organization. 1991.

[15]. Anorlu RI, Odum CU, and Essien EE. Asymptomatic malaria in pregnant women at booking in a primary health care facility in a periurban community in Lagos, Nigeria. Afr J Med Sci. 2001; 30 (suppl): 39-41.

[16]. Agan TU, Ekabua JE, Iklaki CU, Oyo-Ita A and Ibanga I. Prevalence of asymptomatic malaria parasitemia at first antenatal visit in UCTH Calabar. Asian Pacific Journal of Tropical Medicine. 2010; Pp 1-5.

Table 1: Hemoglobin concentration and asymptomatic malaria parasitemia.

\begin{tabular}{|c|c|c|c|c|}
\hline Hemoglobin concentration & \multicolumn{4}{|c|}{ Malaria $\quad$ parasitemia } \\
\hline & Present & $\%$ & Absent & $\%$ \\
\hline$\geq 11 \mathrm{~g} / \mathrm{dl}$ & 77 & 39.7 & 56 & 100.0 \\
\hline $8-10 \mathrm{~g} / \mathrm{dl}$ & 112 & 57.7 & - & \\
\hline $6-8 \mathrm{~g} / \mathrm{dl}$ & 5 & 2.6 & - & \\
\hline$<6 \mathrm{~g} / \mathrm{dl}$ & - & - & - & \\
\hline Total & 194 & 100.0 & 56 & 100.0 \\
\hline
\end{tabular}


Anemia Among Unbooked Parturients With Asymptomatic Malaria Parasitemia At A Tertiary...

Table 2: Parasite density and hemoglobin concentration.

\begin{tabular}{|c|c|c|c|c|c|c|c|c|c|c|}
\hline P. Density & \multicolumn{8}{|c|}{ Hemoglobin $\quad$ Concentration } & $\mathrm{X}^{2}$ & P value \\
\hline & $\geq 11 \mathrm{~g}$ & $\%$ & $8-10 \mathrm{~g}$ & $\%$ & $6-8 \mathrm{~g}$ & $\%$ & $<6 \mathrm{~g}$ & $\%$ & & \\
\hline+ & 77 & 43.8 & 95 & 54.0 & 4 & 2.2 & - & - & & 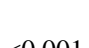 \\
\hline++ & - & & 17 & 94.4 & 1 & 5.6 & - & - & & \\
\hline
\end{tabular}

$+=1-10$ parasites $/ 100$ thick film fields $\quad++=11-100$ parasites/100 thick film fields

Table 3: Maternal hemoglobin concentration.

\begin{tabular}{|l|l|l|}
\hline Hemoglobin concentration & Number(250) & $\%(100)$ \\
\hline$\geq 11 \mathrm{~g}$ & 133 & 53.2 \\
$8-10 \mathrm{~g}$ & 112 & 44.8 \\
$6-8 \mathrm{~g}$ & 5 & 2.0 \\
$<6 \mathrm{~g}$ & - & - \\
\hline
\end{tabular}

